\title{
The Power of AEM and Data Analytics: 20 Years of Teaching MSA at M\&M
}

\author{
Paul G. Kotula ${ }^{1 *}$
}

1. Materials Characterization and Performance, Sandia National Laboratories, Albuquerque, NM, USA.

* Corresponding author: paul.kotula@sandia.gov

Multivariate statistical analysis (MSA) comprises a number of powerful data analytics tools that are well suited to the analysis of spectral images (X-ray, EELS, etc.) [1-2] and more recently to diffraction-image data sets [3-4]. For the spectroscopic data, the number of assumptions (some would call rules today) is kept to a minimum, namely, linear additivity, non-negativity [1], simplicity [2], and a known noise structure (e.g., Poisson) [5-6]. These tools with appropriate assumptions have proven far superior to conventional analysis methods [7-8], both reducing the dimension of large number of noisy observations as well as finding unexpected elements.

Teaching MSA can be difficult as the underlying math can be hard to understand. I've found that it is easier to show how the various MSA tools work and find appropriate application rather than dwell on the underlying algorithms which are all published for those interested [9]. The problem with MSA-based factor analysis methods is that they seek to solve an equation with two unknowns, $\mathrm{D}=\mathrm{CS}^{\mathrm{T}}$, where $\mathrm{D}$ is the raw data, $\mathrm{C}$ is a matrix of component images, $\mathrm{S}$ is a matrix of corresponding spectral shapes and $\mathrm{T}$ denotes the matrix transpose. There are an infinite number of solutions which fit the data equally. By appropriately rotating $\mathrm{C}$ or $\mathrm{S}$ (with corresponding inverse rotation of the complementary matrix) [2] we can generate a solution which, after application of non-negativity, is simple in either the spectral or spatial domain.

Figure 1 shows the MSA of an SEM X-ray spectral image. The sample consists of a number of different composition wires, several with elements in common. The factors in this case have been rotated such that the component images are simple or alternatively high-contrast. The individual component images correspond in this case to chemical phases. This method might give confusing results is several phases were overlapped in transmission as in the AEM. Figure 2 shows the MSA of the same spectral image with the factors rotates such that $\mathrm{S}$ is simple in structure. This provided a more elemental view of the data and is appropriate for cases in the AEM where we have precipitates in a matrix. The precipitates will come out as a separate component with elements unique from the matrix [10].

\section{References:}

[1] PG Kotula, MR Keenan and JR Michael, Microsc Microanal 9 (2003), p. 1.

[2] MR Keenan, Surf. Int. Anal. 41 (2009), p. 79.

[3] LN Brewer, PG Kotula and JR Michael, Ultramicroscopy 108 (2008), p. 567.

[4] AJ Wilkinson et al., Ultramicroscopy 196 (2019), p. 88.

[5] MR Keenan and PG Kotula, Appl. Surf. Sci. 231/232 (2004), p. 240.

[6] MR Keenan and PG Kotula, Surf Int Anal 36 (2004), p. 203.

[7] PG Kotula and MR Keenan (2006).

[8] PG Kotula in "Transmission Electron Microscopy: Diffraction, Imaging, and Spectrometry", ed. CB Carter and DB Williams, 2nd Ed. (Springer, NY), Ch. 2. 
[9] MR Keenan in "Techniques and Applications of Hyperspectral Image Analysis", ed. H Grahn and P Geladi, (John Wiley \& Sons, Chinchester).

[10] Sandia National Laboratories is a multimission laboratory managed and operated by National Technology \& Engineering Solutions of Sandia, LLC, a wholly owned subsidiary of Honeywell International Inc., for the U.S. Department of Energy's National Nuclear Security Administration under contract DE-NA0003525. This paper describes objective technical results and analysis. Any subjective views or opinions that might be expressed in the paper do not necessarily represent the views of the U.S. Department of Energy or the United States Government.

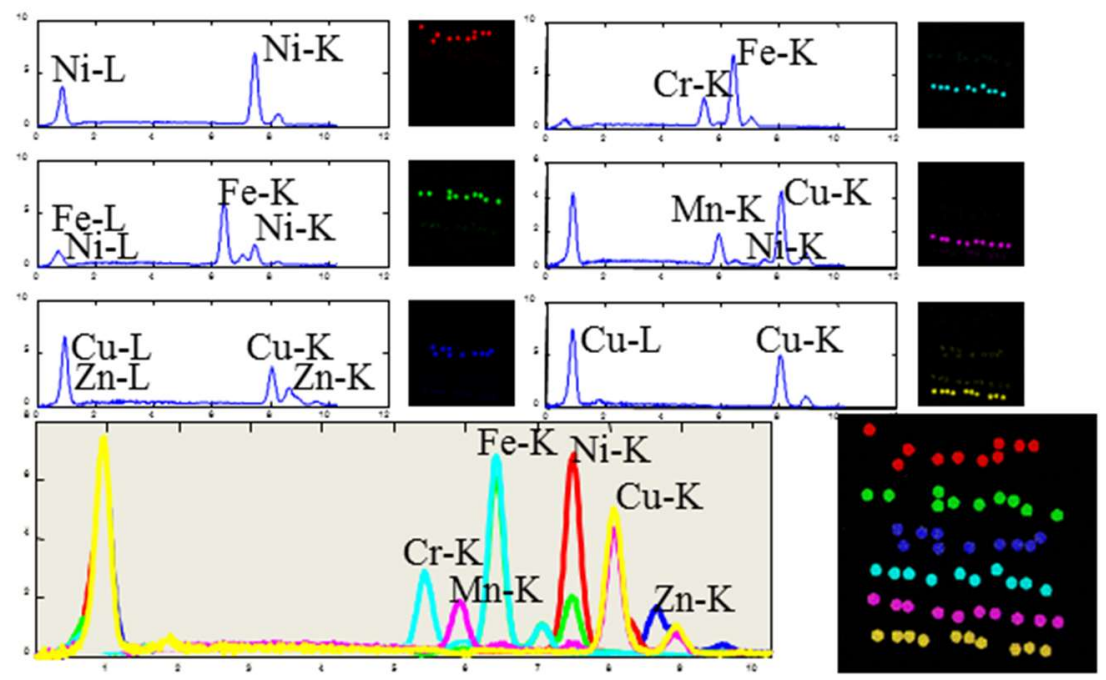

Figure 1. MSA of an X-ray spectral image. The factors are spatially simple as evidenced by the clear breakout by pure color in the overlay. This is often the chemical phase representation of the data.
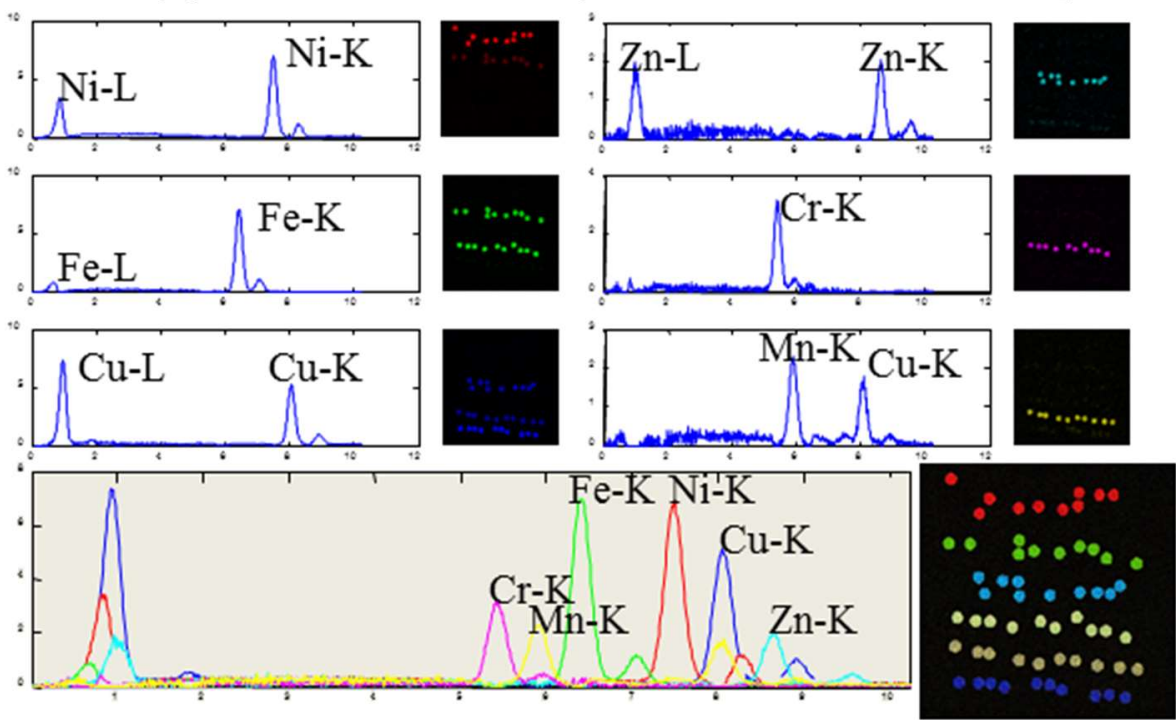

Figure 2. MSA of the same spectral image where the factors are spectrally simple. The color overlay shows mixing of the factors spatially but the spectral factors are simple in structure. 